\title{
Efecto de abonos orgánicos sobre el crecimiento y producción del plátano Hartón (Musa AAB)
}

\section{Effect of organic fertilizers on growth and production of the Harton plantain (Musa AAB)}

JOSÉ L. BARRERA ${ }^{1,2}$

ENRIQUE M. COMBATT ${ }^{1}$

YAN L. RAMÍREZ1

Racimo de plátano Hartón creciendo en el municipio de San Juan de Urabá.

Foto: Y.L. Ramírez

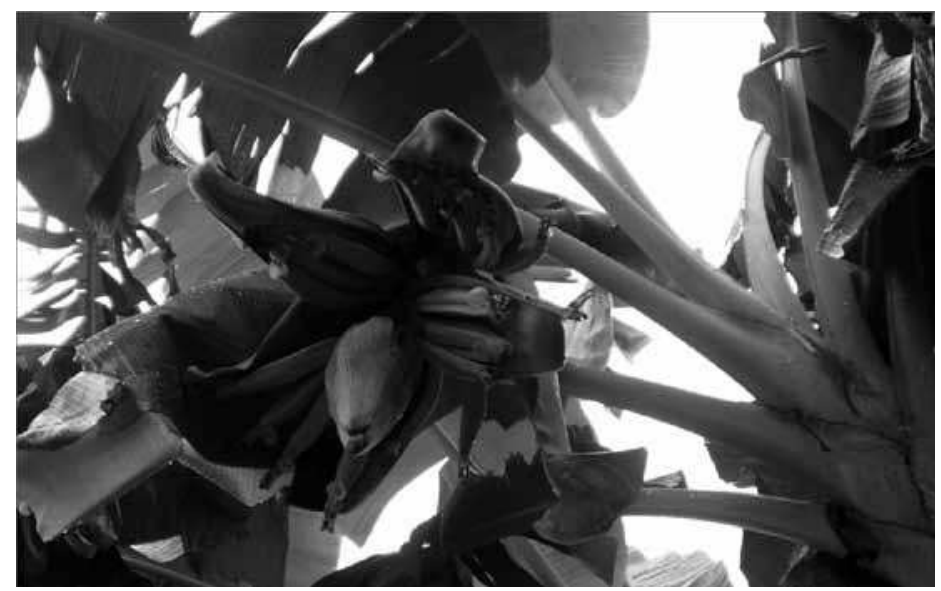

\section{RESUMEN}

Esta investigación se realizó en el municipio San Juan de Urabá (Antioquia), en dos ciclos: 2006-2007 y 20082009, con el objeto de evaluar el efecto de abonos orgánicos sobre el crecimiento y producción del plátano. Los tratamientos fueron distribuidos bajo un diseño en bloques completos al azar, 12 tratamientos y tres repeticiones, la población fue 1.080 plantas, sembradas a $3 \times 3 \mathrm{~m}$ y la unidad experimental estuvo constituida por 30 plantas. Los tratamientos fueron: micorrizas, Bocashi, biol, lombriabono, testigo, químico, micorrizas + Bocashi, micorrizas + Bocashi + biol, micorrizas + lombriabono, micorrizas + lombriabono + biol, micorrizas + biol y micorriza comercial (NN Garcés). La aplicación de los tratamientos fue mensual, 3 meses después de establecido el cultivo (DDE) hasta 9 meses DDE, en las dosis recomendadas, se realizaron las prácticas culturales convencionales en la zona. Para el primer ciclo los abonos orgánicos no influyeron sobre las variables de crecimiento y desarrollo, solo afectaron el peso del racimo, grosor y longitud de la primera mano del racimo y no afectaron el número de dedos y el grosor de la tercera mano, para el segundo ciclo influyeron significativamente en las variables de crecimiento, desarrollo y de producción, no logró influir en el peso del racimo y en el grosor de la tercera mano.

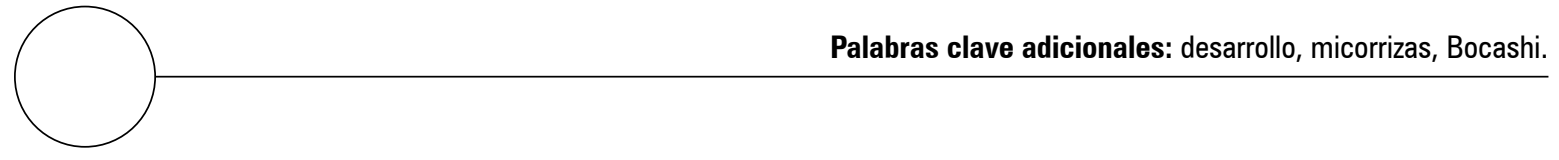

Facultad de Ciencias Agrícolas, Universidad de Córdoba, Montería (Colombia).

2 Autor para correspondencia. jbarrera11@sinu.unicordoba.edu.com; jbarrera11@gmail.com 


\section{ABSTRACT}

This research was conducted in the municipality of San Juan de Urabá (Antioquia) in two cycles: 2006-2007 and 2008-2009, in order to evaluate the effect of organic fertilizers on growth and yield of the plantain. The treatments were distributed under a design of randomized blocks, 12 treatments and three repetitions; the population was 1080 plants planted at $3 \times 3 \mathrm{~m}$, the experimental unit was 30 plants. The treatments were: mycorrhizae, Bocashi, biol, lombriabono, control, chemical, mycorrhizae+Bocashi, mycorrhizae+ Bocashi + biol, mycorrhizae + lombriabono, mycorrhizae + lombriabono + biol, mycorrhizae + biol and commercial mycorrhizae (NN Garcés). The application of the treatments was monthly, established 3 months after planting (MAP) to 9 months MAP, at recommended doses, with the conventional cultural practices of the region. For the first cycle, organic fertilizers did not influence growth and development variables, only affected bunch weight, thickness and length of the first bunch hand and failed to influence the number of fingers or the thickness of the third hand; for the second cycle, organic fertilizers significantly influenced the growth, development and production variables but not bunch weight or the thickness of the third hand.

Additional keywords: development, mycorrhizae, Bocashi.

Fecha de recepción: 02-05-2011

Aprobado para publicación: 21-11-2011

INTRODUCCIÓN

Se estima que del área cultivada en plátano en Colombia, $87 \%$ se encuentra como cultivo tradicional asociado con café, cacao, yuca y frutales, y el restante $13 \%$ está como monocultivo tecnificado. Actualmente cerca de $4 \%$ de la producción nacional de plátano se destina al mercado de exportación, el restante se destina al consumo interno en fresco y una muy pequeña proporción, menos del $1 \%$, se usa como materia prima para la agroindustria nacional. Las regiones del golfo de Urabá y el nororiente del departamento del Magdalena, sobresalen por el grado de especialización que han alcanzado en la producción y exportación de banano y plátano con altos niveles de productividad e integración de los productores y comercializadores, entre otras razones, por las ventajas comparativas de localización y calidad de los suelos con respecto a otras zonas productoras del mundo (MADR, 2009).
El municipio de San Juan de Urabá posee un área de 23.900 ha, de estas, 4.133 están cultivadas en plátano, que equivalen a 17,2\% del área total del municipio, con un rendimiento de $10 \mathrm{t} \mathrm{ha}^{-1} \mathrm{año}^{-1}$ (Anónimo, 2011). Aporta una producción para el mercado nacional de $32.686 \mathrm{t}_{\text {año-1 }}$ y una pro- $^{-}$ ducción de 9.792 t año-1 para el mercado internacional, que entran a los respectivos mercados a través de empresas comercializadoras, las cuales trabajan con exigentes normas de calidad para la fruta de exportación (CCI, 2000).

En cada cosecha se extraen del suelo ciertas cantidades de elementos minerales que hacen que el suelo sea más pobre cuando se cultiva que cuando permanece en barbecho; el suelo bajo cultivos pierde gran parte de su dinámica biológica debido al uso de agroquímicos; a las sales de los fertilizantes inorgánicos; a los altos volúmenes de agua 
de riego, que provocan lixiviación y percolación profunda de elementos nutritivos y lavado de bases, cambiando el $\mathrm{pH}$ del suelo y causando toxicidad; todos estos factores contribuyen a la pérdida de la fertilidad y la biodiversidad del suelo (Orozco, 1999). Los sistemas de producción en plátano de la zona en estudio presentan muchas limitantes para su explotación, entre las que se destacan problemas fitosanitarios, manejo agronómico inadecuado, prácticas culturales inapropiadas, uso irracional de agroquímicos entre otros. El cultivo orgánico en el mundo crece en importancia debido a sus efectos beneficiosos: reduce notablemente el uso de los fertilizantes químicos, mejora los suelos en las propiedades fisicoquímicas y aumenta la microflora favorable. Cuando la materia orgánica es de fácil descomposición, al agregarse al suelo, enriquece al medio con fauna y flora, especialmente con bacterias. Así se activa la descomposición de la materia orgánica nativa del suelo y de la agregada, logrando un beneficio para la nutrición de cultivos (Muñoz, 1994). También otros autores como Bolaños et al. (2003) exponen que la aplicación conjunta de materia orgánica y fertilizante mineral ejerce un efecto positivo sobre el suelo, contribuyendo con el crecimiento y la formación de raíces secundarias y terciarias. Esto a su vez, mejora la capacidad de absorción de nutrientes minerales por las plantas de plátano.

En las zonas productoras de Urabá de gran importancia, no se han realizado estudios que permitan a los productores evaluar estrategias de manejo sostenible de los suelos, utilizando como alternativa abonos orgánicos como sustituto de los fertilizantes químicos ya que el acceso al mercado de los productos orgánicos es cada día más exigente en mercados nacionales e internacionales. En sus estudios, Castillo et al. (1995) y Muñoz (1995) concluyeron que en suelos de mediana a alta fertilidad, el cultivo de plátano no responde a la fertilización química con los elementos mayores. Además, en el país son pocos los reportes de investigación sobre la fertilización orgánica de plátano, que el manejo sostenible del recurso suelo debe incluir todos sus componentes y que existe un desconocimiento de los productores en sistemas de producción limpia u orgánica y sus beneficios al suelo, se planteó este estudio con el objetivo de evaluar la respuesta de la aplicación de abonos orgánicos sobre el crecimiento y producción del cultivo del plátano Hartón en el municipio de San Juan de Urabá (Antioquia).

\section{MATERIALES Y MÉTODOS}

El estudio se realizó durante dos ciclos de producción. El primero entre 2006-2007 y el segundo entre 2008-2009, en la localidad San Juan de Urabá, departamento de Antioquia, situado en el extremo norte del departamento, en la parte baja del río San Juan, localizado en las coordenadas $08^{\circ} 46^{\prime} 17^{\prime \prime} \mathrm{N}$ y $76^{\circ} 31^{\prime} 29^{\prime \prime} \mathrm{W}$, comprende una extensión costera sobre el mar Caribe de 21,5 km. La zona se encuentra a $3 \mathrm{msnm}$, con un temperatura promedio entre los 28 y $30^{\circ} \mathrm{C}$. Fue realizado un muestreo al inicio y al final de la investigación para conocer las características químicas del suelo donde se establecieron los ensayos. Estas muestras se llevaron al Laboratorio de Suelos de la Universidad de Córdoba y fueron procesadas de acuerdo con las metodologías recomendadas por el IGAC (1990). Los tratamientos se distribuyeron en un diseño de bloques completamente al azar, donde se evaluaron 12 tratamientos y tres repeticiones por cada tratamiento. Los tratamientos fueron: micorrizas, Bocashi, biol, lombriabono, testigo, químico, micorrizas + Bocashi, micorrizas + Bocashi + biol, micorrizas + lombriabono, micorrizas + lombriabono + biol, micorrizas + biol y micorriza comercial (NN Garcés) (tabla 1), la población estuvo constituida por 1.080 plantas de plátano del clon Hartón, sembradas a partir de cormos a una distancia de $3 \times 3 \mathrm{~m}$, cada unidad experimental estuvo conformada por 30 plantas, a las cuales se le evaluaron las variables de crecimiento y desarrollo como: número de hojas (emitidas mensualmente), perímetro del pseudotallo $(\mathrm{cm}$, medido mensualmente en la base del tallo a $100 \mathrm{~cm}$ de altura desde la superficie del suelo), 
Tabla 1. Descripción de tratamientos en el municipio San Juan de Urabá (Antioquia).

\begin{tabular}{|c|c|c|c|}
\hline Fuentes de abono & Dosis/planta & $\begin{array}{l}\text { Dosis } \\
\left(\mathrm{kg} \mathrm{ha}^{-1}\right)\end{array}$ & $\begin{array}{c}\text { Dosis } \\
\left(\mathrm{cm}^{3} \mathrm{ha}^{-1}\right)\end{array}$ \\
\hline Micorrizas $^{1}$ & $100 \mathrm{~g}$ & 111.0 & \\
\hline Bocashi & $1.500 \mathrm{~g}$ & 1666.0 & \\
\hline Biol & $833 \mathrm{~cm}^{3}$ al $10 \%$ & & 925.465 \\
\hline Lombriabono & $500 \mathrm{~g}$ & 555,0 & \\
\hline Testigo & 0 & 0 & \\
\hline Químico NPK (15-15-15) & $100 \mathrm{~g}$ & 111,0 & \\
\hline Micorrizas + Bocashi & $100 g+1.500 g$ & $1+2$ & \\
\hline Micorrizas + Bocashi + biol & $100 \mathrm{~g}+1.500 \mathrm{~g}+833 \mathrm{~cm}^{3}$ al $10 \%$ & $1+2+3$ & \\
\hline Micorrizas + lombriabono & $100 g+500 g$ & $1+4$ & \\
\hline Micorrizas + lombriabono + biol & $100 \mathrm{~g}+500 \mathrm{~g}+833 \mathrm{~cm}^{3}$ al $10 \%$ & $1+3+4$ & \\
\hline Micorrizas + biol & $100 \mathrm{~g}+833 \mathrm{~cm}^{3}$ al $10 \%$ & $1+3$ & \\
\hline NN Garcés* & $100 \mathrm{~g}$ & 111,0 & \\
\hline
\end{tabular}

${ }^{1}$ Micorriza comercial.

altura de la planta $(\mathrm{cm}$, medida mensualmente desde base del tallo hasta el punto de inserción de la hoja presente). La aplicación de los tratamientos fue mensual, desde los 3 hasta los 9 meses $\mathrm{DDE}$, en las dosis recomendadas. Durante todo el experimento se realizaron las prácticas culturales convencionales en la zona.

Las variables de producción fueron evaluadas al momento de la cosecha de los racimos: peso bruto del racimo $(\mathrm{kg})$, número de dedos por racimo (unidad), longitud del fruto ( $\mathrm{cm}$, de los dedos centrales de la primera y tercera mano del racimo), perímetro central del fruto $(\mathrm{cm}$, de los dedos centrales de la primera y tercera mano del racimo); todas estas medidas fueron realizadas con una cinta métrica. Los datos obtenidos fueron sometidos a análisis de varianza y a la prueba de comparación de medias Tukey $(P \leq 0,05)$ utilizando el programa estadístico SAS.

\section{RESULTADOS Y DISCUSIÓN}

\section{Número de hojas (primer ciclo)}

No se reportaron efectos significativos entre tratamientos, también se observó que la aplicación de micorrizas combinada con Bocashi o lombriabono estimuló la emisión de hojas (tabla 2).
Estos resultados no coinciden con lo reportado por Jaller y Vargas (2001), cuando aplicaron fertilizantes químicos $\left(250 \mathrm{~kg} \mathrm{ha}^{-1} \mathrm{~N}, 1.200 \mathrm{~kg} \mathrm{ha}^{-1}\right.$ $\mathrm{K}_{2} \mathrm{O}$ ), y encontraron que las plantas en promedio emitieron 38,6 hojas. Igualmente no coinciden con los reportados por Belalcazar (1995) y Barrera y Viera (1993) que al aplicar diferentes fuentes y concentraciones de nutrientes reportaron similares resultados a los de Jaller y Vargas (2001). Lo que posiblemente se relaciona con el nivel de fertilidad de los suelos utilizados en el estudio y a la influencia de variables climáticas, las cuales son ideales, para la siembra del cultivo y a su vez favorecen un normal crecimiento y desarrollo. También estos resultados pueden ser explicados de acuerdo con lo reportado por Belalcazar (1995), quien manifiesta que existe una relación inversa entre el tamaño de la semilla (el cormo) y la emisión de hojas, muy probablemente este factor incidió en mayor emisión de hojas.

\section{Número de hojas (segundo ciclo)}

Las plantas presentaron mayor respuesta $(P \leq 0,01)$ al tratamiento químico con 41,9 hojas, lo cual se debe posiblemente a la mayor solubilidad y disponibilidad de nutrientes que puede aportar esta fuente comparada con las fuentes orgánicas. De igual forma los tratamientos en combinaciones de 
abonos orgánicos favorecieron la emisión de un mayor número de hojas, la aplicación de micorrizas + Bocashi, micorrizas + Bocashi + biol, micorrizas + lombriabono, lombriabono generaron 41,5; 41,1; 41,0 y 40,6 hojas, respectivamente, superando al testigo sin fertilizar; estos tratamientos superaron lo encontrado por Valencia et al. (1994) y Hernández et al. (2007), quienes reportaron tanto en plátano Dominico y Hartón un rango de 36 a 39,6 hojas emitidas totales. Esto demuestra que la combinación de fuentes orgánicas permite un mayor sinergismo en la absorción de nutrientes, y cuando se fertiliza con fuentes individuales solo se logra aportar una menor cantidad de elementos minerales. Esta repuesta se debió, probablemente, al aporte de nutrientes que se originan de las fuentes orgánicas después de un tiempo cuando hay mineralización de las mismas.

\section{Perímetro del pseudotallo (primer y segundo ciclo)}

No se evidenciaron efectos significativos para el primer ciclo de producción, en tanto que para el segundo ciclo el análisis estadístico muestra diferencias significativas $(P \leq 0,01)$, los mejores tratamientos fueron micorrizas + lombriabono con $55,1 \mathrm{~cm}$ y el tratamiento Químico con 51,2 $\mathrm{cm}$ (tabla 2), lo cual demuestra las bondades de la combinación de fuentes de abonos orgánicos. Los valores encontrados en esta investigación fueron superiores a lo reportado por Echeverri (1999) que al aplicar estiércol de bovino en una dosis de 2 kg/planta, 100 g de 18-18-18 al momento de la siembra y $100 \mathrm{~g}$ de urea y cloruro de potasio cada $60 \mathrm{~d}$ durante 4 meses, encontraron un promedio máximo de 48 a $45 \mathrm{~cm}$ de perímetro.

\section{Altura de planta (primer y segundo ciclo)}

En el primer ciclo no se encontraron diferencias significativas, mientras que para el segundo los mejores tratamientos $(P \leq 0,01)$ fueron Bocashi y micorrizas + lombriabono con 169,3 y 168,0 $\mathrm{cm}$. Estos resultados son similares a los reportados por Arcila et al. (2001) quienes evaluaron diferentes sustratos sobre el crecimiento de las plántulas de Dominico-Hartón y encontraron

\begin{tabular}{|c|c|c|c|c|c|c|}
\hline \multirow[b]{2}{*}{ Fuente de abono } & \multicolumn{3}{|c|}{ Primer ciclo } & \multicolumn{3}{|c|}{ Segundo ciclo } \\
\hline & No. hojas & $\begin{array}{l}\text { Perímetro } \\
\text { del tallo } \\
(\mathrm{cm})\end{array}$ & $\begin{array}{l}\text { Altura de } \\
\text { planta }(\mathrm{m})\end{array}$ & No. hojas & $\begin{array}{l}\text { Perímetro del } \\
\text { tallo }(\mathrm{cm})\end{array}$ & $\begin{array}{c}\text { Altura de } \\
\text { planta }(\mathrm{cm})\end{array}$ \\
\hline Micorrizas & 34,3 a & 59,23 a & 202,67 a & 38,7 ef & 45,6 bc & $137,0 \mathrm{bc}$ \\
\hline Bocashi & 37,4 a & 54,2 a & 215,67 a & 39,9 bcde & $49,5 a b$ & 169,3 a \\
\hline Biol. & 37,26 a & 49,23 a & 188,67 a & 40,5 abcde & $45,3 \mathrm{bc}$ & $147,2 \mathrm{ab}$ \\
\hline Lombriabono & 35,23 a & 58,2 a & 225,0 a & 40,6 abcd & $50,5 \mathrm{ab}$ & $150,4 \mathrm{ab}$ \\
\hline Testigo & 34,66 a & $48,9 \mathrm{a}$ & 193,93 a & $38,0 \mathrm{f}$ & $42,4 \mathrm{C}$ & $116,8 \mathrm{c}$ \\
\hline Químico NPK & 36,4 a & 51,2 a & 214,33 a & 41,9 a & $51,2 a b$ & $153,3 \mathrm{ab}$ \\
\hline Micorrizas + Bocashi & 38,33 a & 54,0 a & 218,67 a & $41,1 \mathrm{abc}$ & $47,7 \mathrm{bc}$ & $150,3 \mathrm{ab}$ \\
\hline Micorrizas + Bocashi + biol & 32,93 a & 54,2 a & 216,0 a & $41,0 \mathrm{abcd}$ & $49,2 \mathrm{abc}$ & $155,9 \mathrm{ab}$ \\
\hline Micorrizas + lombriabono & 40,26 a & 60,08 a & 259,33 a & $41,5 \mathrm{ab}$ & $55,1 \mathrm{a}$ & 168,0 a \\
\hline Micorrizas + lombriabono + biol & 38,06 a & 47,63 a & 206,67 a & 40,4 abcde & $48,6 a b c$ & $148,6 \mathrm{ab}$ \\
\hline Micorrizas + biol & 34,16 a & 45,96 a & 206,67 a & 39,3 def & $45,8 \mathrm{bc}$ & $143,9 \mathrm{~b}$ \\
\hline NN Garcés & 36,7 a & 46,9 a & 206,67 a & 39,4 cdef & $46,9 \mathrm{bc}$ & $136,4 \mathrm{bc}$ \\
\hline C.V. & 10,659 & 12,918 & 22,601 & 8,02 & 13,18 & 13,73 \\
\hline $\mathrm{F}$ (tratamientos) & n.s & n.s & n.s & $<0,001$ & $<0,001$ & $<0,001$ \\
\hline
\end{tabular}

Promedios con letras distintas indican diferencia significativa según la prueba de Tukey $(P \leq 0,01)$. 
que la fuente de abono orgánico aplicado contribuyó a un mayor crecimiento en altura y perímetro del pseudotallo.

De manera general, en este ensayo, las fuentes de abonos orgánicos, aportaron los requerimientos nutricionales balanceados $\mathrm{y}$, combinadas con unas buenas prácticas de manejo agronómico, permitieron un adecuado crecimiento en las plantas, comparados con estudios previos para el clon Hartón en la zona de estudio de San Juan de Urabá (Jaller y Vargas, 2001; Combatt et al., 2004).

\section{Peso del racimo}

Se presentaron efectos significativos $(P \leq 0,01)$ entre los tratamientos y los mejores tratamientos fueron el químico y el de micorrizas + lombriabono + biol con 13,5 y 13,4 kg respectivamente (tabla 3). Estos resultados son inferiores a los reportados por Combatt et al. (2004), que aplicando $300 \mathrm{~kg}$ de $\mathrm{N}$ y dosis mayores de 400 kg de $\mathrm{K}$, lograron 25,63 y 25,44 kg de racimo, de forma respectiva. De igual forma, Hernández et al. (2007) encontraron para la variable peso del racimo, valores de $17,74 \mathrm{~kg}$ y $13,05 \mathrm{~kg} / \mathrm{racimo}$ el menor en la zona Sur del Lago de Maracaibo (Venezuela). Es preciso anotar que para el segundo ciclo las plantas estuvieron sometidas a un estrés hídrico provocado por la época seca (atmósfera seca), de manera general la fotosíntesis fue posiblemente afectada primero por la ausencia de agua, lo cual se ve reflejado en el peso del órgano de interés comercial (fruto). En otros estudios, Rodríguez y Rodríguez (1997), sobre el diagnóstico nutricional del plátano, establecieron un rendimiento máximo de más de $15 \mathrm{~kg} / \mathrm{racimo}$ en plantas de plátano Dominico. Sin embargo los resultados permiten inferir que las fuentes orgánicas y química evaluadas superaron al testigo absoluto, lo cual refleja que estas aportan nutrientes necesarios a la planta, y que la mezcla de fuentes permite mayor sinergismo, igualando las fuentes químicas.

\section{Número de dedos por racimo}

Se reportaron efectos significativos $(P \leq 0,01)$ para esta variable y los mejores tratamientos fueron el químico, micorrizas + Bocashi + biol y micorrizas + lombriabono con 35,0, 30,33 y 30,33 dedos por racimo respectivamente (tabla 3 ). Espinosa et al. (1998) aseguran que la expresión de los números de dedos es codificada genéticamente al momento de la diferenciación floral y solamente es alterada cuando se presentan condiciones adversas antes de que ocurra dicha diferenciación. Los resultados obtenidos, para esta variable son inferiores a los observados por Jaller y Vargas (2001) debido posiblemente, a la poca oferta hídrica en el segundo semestre, coincidiendo con la época seca; este factor impide, posiblemente, la expresión del potencial genético de la planta.

\section{Longitud del dedo central de la primera mano del racimo}

En cuanto a esta variable es de suma importancia como indicador de la calidad del fruto, se reportaron diferencias significativas entre los tratamientos $(P \leq 0,01)$ y los mejores tratamientos fueron micorrizas + Bocashi + biol y el lombriabono, con 29,6 y $29,2 \mathrm{~cm}$, respectivamente (tabla 3). Estos resultados son similares a los reportados por Combatt et al. (2004), quienes al aplicar dosis de $300 \mathrm{~kg} \mathrm{ha}^{-1}$ de $\mathrm{N}$ y $1.200 \mathrm{~kg} \mathrm{ha}^{-1}$ de $\mathrm{K}_{2} \mathrm{O}$ obtuvieron $29,6 \mathrm{~cm}$.

\section{Longitud del dedo central de la tercera mano del racimo}

Se presentaron diferencias significativas $(P \leq 0,01)$, los mayores valores se encontraron con la aplicación de micorrizas + Bocashi + biol, y el lombriabono con 27,9 y 27,6 cm, respectivamente (tabla 3). Estos resultados confirmaron que las aplicaciones de abonos orgánicos suplieron las necesidades para un buen llenado de fruto, alcanzando los estándares de calidad tipo exportación (CCI, 2000). 


\begin{tabular}{|c|c|c|c|c|c|c|}
\hline \multirow[b]{2}{*}{ Fuentes de abono } & \multicolumn{6}{|c|}{ Primer ciclo } \\
\hline & $\begin{array}{c}\text { Peso } \\
\text { racimo }(\mathrm{kg})\end{array}$ & $\begin{array}{l}\text { Número } \\
\text { dedos }\end{array}$ & $\begin{array}{l}\text { Longitud } \\
\text { dedo } \\
\text { primera } \\
\text { mano }(\mathrm{cm})\end{array}$ & $\begin{array}{c}\text { Longitud } \\
\text { dedo tercera } \\
\text { mano }(\mathrm{cm})\end{array}$ & $\begin{array}{l}\text { Perímetro } \\
\text { dedo } \\
\text { primera } \\
\text { mano }(\mathrm{cm})\end{array}$ & $\begin{array}{l}\text { Perímetro } \\
\text { dedo } \\
\text { tercera } \\
\text { mano }(\mathrm{cm})\end{array}$ \\
\hline Micorrizas & $11,00 \mathrm{ab}$ & 27,00 a & $29,30 \mathrm{abc}$ & 30,66 abc & $21,70 a b$ & 21,06 a \\
\hline Bocashi & $11,00 \mathrm{ab}$ & 27,30 a & 29,56 abc & $31,30 \mathrm{abc}$ & $23,03 \mathrm{ab}$ & 21,66 a \\
\hline Biol. & $11,88 \mathrm{ab}$ & 28,60 a & $28,30 \mathrm{c}$ & $26,00 \mathrm{c}$ & $25,46 \mathrm{ab}$ & 23,63 a \\
\hline Lombriabono & $11,21 a b$ & 28,30 a & 30,66 abc & 33,73 a & $23,00 \mathrm{ab}$ & 22,96 a \\
\hline Testigo & $8,10 \mathrm{~b}$ & 20,00 a & $28,36 \mathrm{c}$ & 26,66 bc & $20,00 \mathrm{c}$ & 20,06 a \\
\hline Químico NPK & $12,36 \mathrm{ab}$ & 31,00 a & 32,80 a & $32,53 \mathrm{ab}$ & 26,60 a & 24,73 a \\
\hline Micorrizas + Bocashi & $11,48 a b$ & 27,60 a & $31,33 a b c$ & $31,03 a b c$ & 26,36 a & 24,06 a \\
\hline Micorrizas + Bocashi + biol & $12,63 a b$ & 28,00 a & 29,76 abc & $28,93 \mathrm{abc}$ & $24,16 a b$ & 24,13 a \\
\hline Micorrizas + lombriabono & $12,83 \mathrm{ab}$ & 28,00 a & $29,73 \mathrm{abc}$ & 27,16 abc & $23,00 \mathrm{ab}$ & 22,00 a \\
\hline Micorrizas + lombriabono + biol & 13,50 a & 30,30 a & $32,23 \mathrm{ab}$ & 29,26 abc & $25,30 \mathrm{ab}$ & 23,80 a \\
\hline Micorrizas + biol & $11,50 \mathrm{ab}$ & 28,00 a & 28,66 bc & $28,13 \mathrm{abc}$ & $24,43 a b$ & 21,93 a \\
\hline NN Garcés & $10,71 \mathrm{ab}$ & 20,33 a & $27,93 \mathrm{c}$ & 27,66 abc & $21,66 \mathrm{ab}$ & 24,00 a \\
\hline CV (\%) & 12,250 & 5,911 & 3,895 & 6,717 & 7,17 & 7,21 \\
\hline $\mathrm{F}$ (Tratamientos) & $<0,001$ & ns & $<0,001$ & $<0,001$ & $<0,001$ & ns \\
\hline \multicolumn{7}{|c|}{ Segundo ciclo } \\
\hline Micorrizas & 10,0 e & $27,43 b$ & $27,93 \mathrm{~b}$ & $26,70 \mathrm{ab}$ & 17,73 a & $13,66 a b$ \\
\hline Bocashi & $11,16 \mathrm{~cd}$ & $28,33 \mathrm{~b}$ & $28,33 \mathrm{ab}$ & $25,66 \mathrm{ab}$ & 16,66 a & $13,73 \mathrm{ab}$ \\
\hline Biol. & $11,36 \mathrm{~cd}$ & $29,00 \mathrm{~b}$ & $28,10 \mathrm{~b}$ & $26,76 \mathrm{ab}$ & 17,60 a & $13,63 \mathrm{ab}$ \\
\hline Lombriabono & $11,20 \mathrm{~cd}$ & $29,33 \mathrm{~b}$ & 29,26 ab & $27,60 \mathrm{ab}$ & 17,96 a & $14,73 a b$ \\
\hline Testigo & $5,36 \mathrm{f}$ & $17,00 \mathrm{c}$ & $22,36 \mathrm{c}$ & 19,66 c & $13,76 \mathrm{~b}$ & $12,36 \mathrm{~b}$ \\
\hline Químico NPK & 13,50 a & 35,00 a & $29,00 a b$ & $27,43 a b$ & 18,73 a & 15,66 a \\
\hline Micorrizas + Bocashi & $11,16 \mathrm{~cd}$ & $28,76 \mathrm{~b}$ & $27,83 \mathrm{~b}$ & $25,93 \mathrm{ab}$ & 16,76 a & 15,06 a \\
\hline Micorrizas + Bocashi + biol & $12,30 \mathrm{bc}$ & $30,33 \mathrm{~b}$ & 29,66 a & 27,96 a & 18,43 a & $14,83 a b$ \\
\hline Micorrizas + lombriabono & $12,63 \mathrm{ab}$ & $30,33 \mathrm{~b}$ & $28,46 a b$ & $26,43 a b$ & 17,13 a & $13,46 a b$ \\
\hline Micorrizas + lombriabono + biol & $13,40 a b$ & $29,0 \mathrm{~b}$ & $28,76 \mathrm{ab}$ & $27,50 \mathrm{ab}$ & 18,00 a & 15,56 a \\
\hline Micorrizas + biol & 10,80 de & 26,66 b & $29,10 \mathrm{ab}$ & $27,33 \mathrm{ab}$ & $18,10 \mathrm{a}$ & $14,90 \mathrm{ab}$ \\
\hline NN Garcés & 10,56 de & $27,33 \mathrm{~b}$ & $27,93 \mathrm{~b}$ & $25,10 \mathrm{~b}$ & 18,33 a & $14,43 a b$ \\
\hline CV (\%) & 3,52 & 5,28 & 1,80 & 3,59 & 4,06 & 5,99 \\
\hline F (Tratamientos) & $<0,001$ & $<0,001$ & $<0,001$ & $<0,001$ & $<0,001$ & $<0,001$ \\
\hline
\end{tabular}

Promedios con letras distintas indican diferencia significativa según la prueba de Tukey $(P \leq 0,01)$.

\section{Perímetro del dedo central de la primera mano del racimo}

El análisis de varianza indicó diferencias estadísticas entre los tratamientos $y$ los mejores fueron el químico y el de micorrizas + Bocashi + biol con 18,7 y $18,4 \mathrm{~cm}$ (tabla 3). Pese a la falta de agua que es fundamental para el llenado de la fruta, el clon Hartón responde muy bien a las aplicaciones de abonos orgánicos, ya que aportaron los nutrientes necesarios para alcanzar un llenado adecuado de la fruta, y a la vez alcanza- 
ron los rangos exigidos por las comercializadoras de fruta para exportación.

\section{Perímetro del dedo central de la tercera mano del racimo}

No se encontraron diferencias estadísticas $(P>0,01)$ entre los tratamientos, el químico y el de micorrizas + lombriabono + biol con 15,6 y $15,5 \mathrm{~cm}$ tendieron a ser los más grandes (tabla 3). Estos resultados son similares a los reportados por Barrera et al. (2007), quienes evaluaron la contribución del desmame y embolso sobre la producción y calidad del plátano Hartón. Esta variable también alcanzó los rangos exigidos por las comercializadoras de fruta para exportación.

\section{CONCLUSIONES}

Los abonos orgánicos no afectaron las variables de crecimiento y desarrollo en el primer ciclo; sin embargo, para el segundo ciclo, lograron influir significativamente. Los abonos orgánicos incrementaron el peso del racimo, longitud de los dedos de la primera y tercera mano y el grosor de los dedos de la primera mano, pero no lograron afectar positivamente el número de dedos por racimo y el grosor de los dedos de la tercera mano. La aplicación de micorrizas + Bocashi + biol incidió positivamente en la mayoría de las variables de crecimiento, desarrollo y producción de plátano Hartón en los dos ciclos de evaluación.

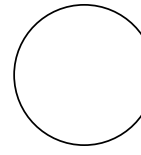

Anónimo. 2011. Municipio San Juan de Urabá En: http:// sanjuandeuraba-antioquia.gov.co/nuestromunicipio.shtml? apc $=$ myxx-1-\&m=f; consulta: marzo de 2011.

Arcila, M., J. Valencia, S. Belalcázar y J. Morales. 2001. Efecto del desmane sobre la calidad y producción del híbrido de plátano FHIA 2. pp. 446-449. En: Memorias XV Reunión Internacional ACORBAT, Asociación de Bananeros de Colombia, Medellín, Colombia.

Belalcázar, S. 1991. El cultivo del plátano en el trópico. Manual de asistencia técnica No. 50. Comité Departamental de Cafeteros del Quindío, Federación Nacional Cafeteros de Colombia, Armenia, Colombia.

Barrera, J. y E. Viera. 1993. Respuesta del cultivo del plátano Hartón a varios niveles y épocas de fertilización potásica en un suelo Tepic Eutropet del Municipio de Tierralta-Córdoba. Trabajo de grado. Facultad de Ciencias Agrícolas, Universidad de Córdoba, Montería, Colombia.

Barrera, J., D. Vergara y O. Marín. 2007. Contribución del desmane y embolse del racimo a la producción y calidad del plátano hartón. Agron. Colomb. 15(1), 39-44.
REFERENCIAS BIBLIOGRÁFICAS

Bolaños, M., H. Morales y L. Celis. 2003. Fertilización (orgánica-química) y producción de 'Dominico hartón'. Infomusa 12, 36-45.

Castillo, L.E., S.C. Belalcázar, J.A. Valencia, M.I. Arcila, J. Espinosa y A. González. 1995. Evaluación de niveles de NPK sobre el crecimiento y la producción del clon de plátano 'Dominico hartón' (Musa AAB Simmonds). pp. 129-141. En: Mejoramiento de la producción del cultivo de plátano. Corpoica, Armenia, Colombia.

Combatt, E.M.; G. Martínez y J.L. Barrera. 2004. Efecto de la interacción de $\mathrm{N}$ y $\mathrm{K}$ sobre las variables de rendimiento del cultivo de plátano (Musa AAB Simmonds) en San Juan de Urabá. Antioquia 9(1), 5-13

CCI. 2000. Acuerdo de competitividad de la cadena productiva del plátano en Colombia. Corporación Colombia Internacional, Bogotá.

Echeverry, E. 1999. Fertilización orgánica vs. fertilización inorgánica de plátano cachaco común en Colombia. Infomusa 10, 7-10.

Espinosa, J., S. Belalcázar, A. Chacón y D. Suarez. 1998. Fertilización del plátano en altas densidades. pp. 79 80. En: Memorias Seminario internacional sobre la producción de plátano. Armenia, Colombia. 
Hernández, Y., M. Marín y J. García. 2007. Respuesta en el rendimiento del plátano (Musa AAB) cv. Hartón en función de la nutrición mineral y su ciclo fenológico. Parte I. Crecimiento y producción. Agron. Colomb. 24(4), 607-626.

IGAC. 1990. Métodos analíticos del laboratorio de suelos. $5 a$ ed. Instituto Geográfico Agustín Codazzi, Bogotá.

Jaller, R. y M. Vargas. 2001. Respuesta del cultivo del plátano (Musa AAB) Simonds a la interacción de N, P, $\mathrm{K}$, aplicado en diferentes dosis en el municipio de San Juan de Urabá-Antioquia. Trabajo de grado. Facultad de Ciencias Agrícolas, Universidad de Córdoba, Montería, Colombia.

MADR. 2009. La cadena de plátano en Colombia. Una mirada global de su estructura y dinámica. Documento de trabajo No. 102. Ministerio de Agricultura y Desarrollo Rural, Bogotá.

Muñoz, R. 1994. Los abonos orgánicos y su uso en la agricultura. pp. 293-304. En: Silva M., F. (ed.). Fertilidad de suelos, diagnóstico y control. Sociedad Colombiana de la Ciencia del Suelo, Bogotá.

Muñoz, R. 1995. Fertilización de cultivos en clima medio. Monómeros Colombo Venezolanos, Barranquilla, Colombia. pp. 195-204.

Orozco, J. 1999. Fertilizantes orgánicos y su aplicación en el cultivo del banano. pp. 82-88. En: Memorias del Taller Internacional Producción de Banano Orgánico y Ambientalmente Amigable. EARTH, Guacimo, Costa Rica.

Rodríguez, V. y O. Rodríguez. 1997. Normas foliares DRIS para el diagnóstico nutricional del plátano (Musa AAB) subgrupo plátano cv. Hartón. Revista Facultad de Agronomía-Universidad de Zulia 14, 285-296.

Valencia, A., L. Castillo, S. Belalcázar y M. Arcila. 1994. Efectos de los diferentes niveles de N, P, K, sobre el crecimiento y la producción de plátano (Musa AAB Simmonds). pp. 757-778. En: Memorias Reunión XI ACORBAT, San José, Costa Rica. 